\title{
Determination of neutrino mixing parameters after SNO oscillation evidence
}

\author{
P. Aliani, ${ }^{1, *}$ V. Antonelli, ${ }^{1, \dagger}$ R. Ferrari, ${ }^{1, \hbar}$ M. Picariello, ${ }^{1, \S}$ and E. Torrente-Lujan ${ }^{1,2,3, \|}$ \\ ${ }^{1}$ Dipartimento di Fisica, Università di Milano, and INFN Sezione Milano, Via Celoria 16, Milano, Italy \\ ${ }^{2}$ Departamento de Fisica Teorica C-XI, Universidad Autonoma de Madrid, 28049 Madrid, Spain \\ ${ }^{3}$ CERN TH-Division, CH-1202 Geneve, Switzerland \\ (Received 7 August 2002; published 24 January 2003)
}

\begin{abstract}
An updated analysis of all available neutrino oscillation evidence in solar experiments (SK day and night spectra, global rates from Homestake, SAGE, and GALLEX) including the latest SNO CC and NC data is presented. Assuming that the shape of the SNO CC energy spectrum is undistorted and using the information provided by SNO we obtain, for the fraction of electron neutrinos remaining in the solar beam at energies $\gtrsim 5$ $\mathrm{MeV}, \phi_{\mathrm{CC}} / \phi_{\mathrm{NC}}=0.34_{-0.04}^{+0.05}$, which is nominally many standard deviations away from the standard value. The fraction of oscillating neutrinos into active ones is computed to be $\left(\Phi_{\mathrm{NC}}-\Phi_{\mathrm{CC}}\right) /\left(\Phi_{\mathrm{SSM}}-\Phi_{\mathrm{CC}}\right)=0.92_{-0.20}^{+0.39}$, nearly $5 \sigma$ deviations from the pure sterile oscillation case. The data are still compatible with an important fraction of sterile component in the solar beam (up to $20 \%-30 \%$ of the total). In the framework of two active neutrino oscillations we determine individual neutrino mixing parameters and their errors in the region of no spectrum distortion $\left(\Delta\left\langle T_{e}\right\rangle<1 \%\right)$; we obtain $\Delta m^{2}=4.5_{-1.4}^{+2.7} \times 10^{-5} \mathrm{eV}^{2}, \tan ^{2} \theta=0.40_{-0.08}^{+0.10}$. These parameters are in agreement with those ones of the best $\chi^{2}$ solution in the LMA region.
\end{abstract}

DOI: 10.1103/PhysRevD.67.013006

PACS number(s): 26.65.+t, 14.60.Pq

\section{INTRODUCTION}

The observation of neutral current neutrino interactions on deuterium in the SNO experiment has been recently presented $[1,2]$. Using the neutral current (NC), elastic scattering (ES), and charged current (CC) reactions and assuming the ${ }^{8} \mathrm{~B}$ neutrino shape predicted by the standard solar model (SSM), the electron and active nonelectron neutrino component of the solar flux at high energies $(\geq 5 \mathrm{MeV})$ are obtained. The nonelectron component is found to be $\sim 5 \sigma$ greater than zero, the standard prediction, thus providing the strongest evidence so far for flavor oscillation in the neutral lepton sector: the agreement of the total flux, provided by the NC measurement with the expectations implies as a byproduct the confirmation of the validity of the SSM [3-5].

The SNO experiment measures the ${ }^{8} \mathrm{~B}$ solar neutrinos via the following reactions [6-9]: (1) charged current (CC): $\nu_{e}$ $+d \rightarrow 2 p+e^{-}$, (2) elastic scattering (ES): $\nu_{x}+e^{-} \rightarrow \nu_{x}$ $+e^{-}$, and (3) neutral current (NC): $\nu_{x}+d \rightarrow p+n+\nu_{x}$. The first reaction is sensitive exclusively to electron neutrinos. The second, the same as the one used at Super-Kamiokande (SK), is instead sensitive, with different efficiencies, to all flavors. Finally the NC reaction is equally sensitive to all active neutrino species.

The results presented recently by SNO on solar neutrinos [10] confirm and are consistent with previous evidence from SK and the rest of the solar neutrino experiments [11$13,14]$. The CC, ES, and NC global and day and night fluxes presented in Refs. [1,2], summarized in Table I, are derived under the assumption that the ${ }^{8} \mathrm{~B}$ spectral shape is not dis-

\footnotetext{
*Email address: paola.aliani@cern.ch

†Email address: vito.antonelli@mi.infn.it

‡Email address: ruggero.ferrari@mi.infn.it

${ }^{\S}$ Email address: marco.picariello@mi.infn.it

"Email address: torrente@cern.ch
}

torted from the SSM prediction. With this assumption the SNO Collaboration checks the hypothesis of nonoscillation, or zero $\phi_{\mu+\tau}$ flux.

It would be advantageous to use this assumption of nondistortion for several reasons. Not only in general terms of simplicity and logical economy but also because with it a much higher statistical accuracy and power of prediction can be obtained (compare the total NC fluxes obtained with and without the distortion hypothesis obtained in [1]). Using these fluxes is appropriate for the calculation of constraints on mixing parameters in theoretical models where such spectrum distortion is negligible, this is true in particular for ample regions of oscillation space favored by all previous data (with or without previous SNO CC data).

Usually, the best fit to the data has been routinely obtained in the large mixing angle (LMA) region (see [15] and refer-

TABLE I. Summary of data used in this work. The observed signal $\left(S_{\mathrm{SSM}}\right)$ and ratios $S_{\text {Data }} / S_{\mathrm{SSM}}$ with respect to the BPB2001 model are reported. The SK and SNO rates are in $10^{6} \mathrm{~cm}^{-2} \mathrm{~s}^{-1}$ units. The $\mathrm{Cl}$, SAGE, and GNO-GALLEX measurements are in SNU units. In this work we use the combined results of SAGE and GNO-GALLEX: $S_{\mathrm{Ga}} / S_{\mathrm{SSM}}(\mathrm{Ga} \equiv \mathrm{SAGE}+\mathrm{GALLEX}+\mathrm{GNO})$ $=0.579 \pm 0.050$. The $\mathrm{SSM}^{8} \mathrm{~B}$ total flux is taken from the BPB2001 model [4]: $\phi_{\nu}\left({ }^{8} \mathrm{~B}\right)=5.05\left(1_{-0.16}^{+0.20}\right) \times 10^{6} \mathrm{~cm}^{-2} \mathrm{~s}^{-1}$.

\begin{tabular}{lcc}
\hline \hline Experiment [Ref.] & $S_{\mathrm{SSM}}$ & $S_{\text {Data }} / S_{\mathrm{SSM}}( \pm 1 \sigma)$ \\
\hline SK $(1258 \mathrm{~d})[38]$ & $2.32 \pm 0.03 \pm 0.08$ & $0.451 \pm 0.011$ \\
Cl [41] & $2.56 \pm 0.16 \pm 0.16$ & $0.332 \pm 0.056$ \\
SAGE [14,35] & $67.2 \pm 7.0 \pm 3.2$ & $0.521 \pm 0.067$ \\
GNO-GALLEX [36,37] & $74.1 \pm 6.7 \pm 3.5$ & $0.600 \pm 0.067$ \\
SNO data [1,2]: & & \\
SNO-CC & $1.76 \pm 0.06 \pm 0.09$ & $0.348 \pm 0.020$ \\
SNO-ES & $2.39 \pm 0.24 \pm 0.12$ & $0.473 \pm 0.053$ \\
SNO-NC & $5.09 \pm 0.44 \pm 0.45$ & $1.008 \pm 0.125$ \\
$A_{e}\left(A_{\mathrm{TOT}} \equiv 0\right)[2]$ & $+0.070 \pm 0.052$ & \\
\hline \hline
\end{tabular}


ences therein), the qualitative explanation for that being the fact that just in that region the observed rather undistorted SK spectrum can be optimally adjusted. The spectrum distortion of the oscillating solutions for SNO in the LMA region has been explicitly found to be negligible [16]. Quantitatively, the main information content of the shape of the observable spectrum is summarized by the first moment of the distribution, the average spectrum energy. For the SSM case and in the absence of oscillations, it is found in Ref. [16] that the average kinetic energy $\left\langle T_{e}\right\rangle=7.658$ $\pm 0.006 \mathrm{MeV}$. This has to be compared with the expected value for a typical, nondistorting, LMA oscillating solution $\left\langle T_{e}\right\rangle=7.654 \mathrm{MeV}$ and with the far values of the distorted small mixing angle (SMA) (7.875 MeV) and vacuum oscillation (VAC) $(8.361 \mathrm{MeV})$ solutions.

In this work we present an up-to-date analysis of all available solar neutrino evidence including the latest SNO results in the most simple framework. First we will reobtain some model independent results which put in a quantitative basis the extent of the deviations with respect to the standard nonoscillating case and the relative importance of active/ sterile oscillations. Second we will obtain allowed areas in parameter space in the framework of active two neutrino oscillations from a standard statistical analysis. Individual values for $\Delta m^{2}$ and $\tan ^{2} \theta$ with error estimation will be obtained from the analysis of marginal likelihoods. In this statistical analysis we include all available data from SK, Homestake, and gallium experiments. From SNO we include the latest results on global day and night fluxes, we make use in particular of (a) the total ${ }^{8} \mathrm{~B}$ flux as measured by the NC reaction and (b) the electron neutrino day-night global asymmetry. The main conclusion of our analysis to be presented below is that it is already possible to determine at present active two neutrino oscillation parameters with relatively good accuracy.

\section{SOME MODEL INDEPENDENT RESULTS}

Different quantities can be defined in order to make the evidence for disappearance and appearance of the neutrino flavors explicit. Letting alone the SNO data, from the three fluxes measured by SNO it is possible to define two useful ratios, deviations of these ratios with respect to their standard value are powerful tests for the occurrence of new physics. Here we compute the values for $\phi_{\mathrm{CC}} / \phi_{\mathrm{ES}}$ and $\phi_{\mathrm{CC}} / \phi_{\mathrm{NC}}$ being specially careful with the treatment of the correlations on the uncertitudes, the inclusion or not of these correlations can affect significantly the results for these ratios (see Table II in Ref. [1] for a complete list of systematical errors). For the first ratio, from the value from SNO rates [1] we obtain

$$
\frac{\phi_{\mathrm{CC}}}{\phi_{\mathrm{ES}}}=0.73_{-0.07}^{+0.10}
$$

a value which is $\sim 2.7 \sigma$ away from the no-oscillation expectation value of one. The ratio of $\mathrm{CC}$ and $\mathrm{NC}$ fluxes gives the fraction of electron neutrinos remaining in the solar neutrino beam, our value is

$$
\frac{\phi_{\mathrm{CC}}}{\phi_{\mathrm{NC}}}=0.34_{-0.04}^{+0.05}
$$

this value is nominally many standard deviations $(\sim 13 \sigma)$ away from the standard model case [16].

Finally, if in addition to SNO data we consider the flux predicted by the solar standard mode one can define, following Ref. [17], the quantity $\sin ^{2} \alpha$, the fraction of "oscillation neutrinos which oscillated into active ones," again using the SNO data and fully applying systematic correlations (see Table II in Ref. [1]), we find the following result:

$$
\sin ^{2} \alpha=\frac{\phi_{\mathrm{NC}}-\phi_{\mathrm{CC}}}{\phi_{\mathrm{SSM}}-\phi_{\mathrm{CC}}}=0.92_{-0.20}^{+0.39} .
$$

The SSM flux is taken as the ${ }^{8} \mathrm{~B}$ flux predicted in Ref. [4]. Note that, although consistent with it, this result differs significantly from the number obtained in Ref. [17], this is due to the introduction of systematic correlations in our calculation. The central value is clearly below one (only active oscillations): the fraction of sterile neutrinos is $\cos ^{2} \alpha$ $\lessgtr 0.28(1 \sigma)$. Although electron neutrinos are still allowed to oscillate into sterile neutrinos the hypothesis of transitions to only sterile neutrinos is rejected at nearly $5 \sigma$, this significance would be reduced if we consider applying a one-sided analysis to avoid nonphysical values.

\section{METHODS AND STATISTICAL PROCEDURES}

The computation of the neutrino oscillation probabilites in solar and earth matter and of the expected signal in each experiment follows the standard methods found in the literature $[15,17-24]$. We solve numerically, as is explained in detail in Ref. [15], the neutrino evolution equations for all the oscillation parameter space. The survival probabilities for an electron neutrino, produced in the Sun, to arrive at the Earth are calculated in three steps. The propagation from the production point to the Sun's surface is computed numerically in all the parameter ranges using the electron number density $n_{e}$ given by the Bahcall-Pinsonneault-Basu 2001 (BPB2001) model [4] averaging over the production point. The propagation in vacuum from the Sun's surface to the Earth is computed analytically. The averaging over the annual variation of the orbit is also exactly performed using simple Bessel functions. To take the earth matter effects into account, we adopt a spherical model of the earth density and chemical composition. In this model, the earth is divided in 11 radial density zones [25], in each of which a polynomial interpolation is used to obtain the electron density. The composition of the neutrino propagation in the three different regions is performed exactly using an evolution operator formalism [24]. The final survival probabilities are obtained from the corresponding (nonpure) density matrices built from the evolution operators in each of these three regions. The night quantities are obtained using appropriate weights which depend on the neutrino impact parameter and the sagitta distance from neutrino trajectory to the Earth's center, for each detector's geographical location.

The expected signal in each detector is obtained by con- 

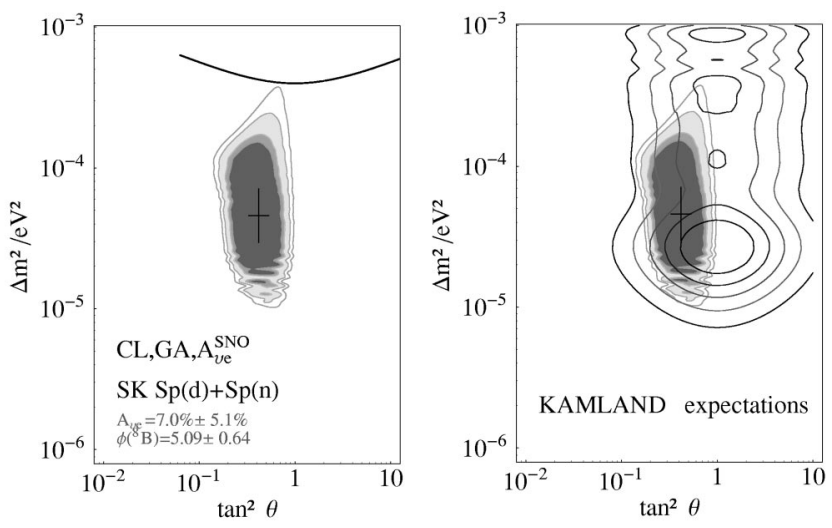

FIG. 1. (Left) Allowed areas in the two neutrino parameter space. The point with error bars corresponds to the best results from fit to marginal likelihoods. The colored areas are the allowed regions at $90 \%, 95 \%, 99 \%$, and $99.7 \%$ C.L. relative to the absolute minimum. The region above the upper thick line is excluded by the reactor experiments [42]. (Right) Superimposed to the figure on the right, KamLAND constant signal contours normalized to the nonoscillation expectation. Contours, from inside to outwards, respectively, at $0.4,0.5,0.6,0.7$, and 0.8 .

voluting neutrino fluxes, oscillation probabilities, neutrino cross sections, and detector energy response functions. We have used neutrino-electron elastic cross sections which include radiative corrections [26]. Neutrino cross sections on deuterium needed for the computation of the SNO measurements are taken from [27]. Detector effects are summarized by the respective response functions, obtained by taking into account both the energy resolution and the detector efficiency. The resolution function for SNO is that given in $[1,2,28]$. We obtained the energy resolution function for SK using the data presented in [29-31]. The effective threshold efficiencies, which take into account the live time for each experimental period, are incorporated into our simulation program. They are obtained from [32].

The statistical significance of the neutrino oscillation hypothesis is tested with a standard $\chi^{2}$ method which is explained in detail in Ref. [15]. Our present analysis is based on the consideration of the following $\chi^{2}$ quantity made of three well differentiated pieces:

$$
\chi^{2}=\chi_{\text {glob }}^{2}+\chi_{\text {spec-sk }}^{2}+\chi_{\text {SNO }}^{2}
$$

The contribution of SNO to the $\chi^{2}$ is given by

$$
\chi_{\mathrm{SNO}}^{2}=\left(\frac{\alpha-\alpha^{\mathrm{th}}}{\sigma_{\alpha}}\right)^{2}+\left(\frac{A_{e}^{\mathrm{th}}-A_{e}^{\mathrm{exp}}}{\sigma_{A}}\right)^{2} .
$$

We have introduced the flux normalization factor $\alpha$ with respect to the SNO NC flux whose central and error values are given in Table I. This flux normalization will be used below as a scale factor for the SK spectrum. The quantity $A_{e}$ is the asymmetry on the day and night electron neutrino rates extracted from the SNO CC, ES, and NC data, imposing the condition $A_{\text {tot }}=A_{e+\mu \tau} \equiv 0$, as predicted by active-only models [2]. The strong anticorrelation of $A_{e}$ and $A_{\text {tot }}$ makes the imposition of this constraint useful, thus reducing greatly the uncertainties with respect to the raw $A_{\mathrm{CC}}$.

The definition of the $\chi_{\text {glob }}^{2}$ function is the following:

$$
\chi_{\text {glob }}^{2}=\left(\mathbf{R}^{\text {th }}-\mathbf{R}^{\exp }\right)^{T}\left(\sigma^{2}\right)^{-1}\left(\mathbf{R}^{\text {th }}-\mathbf{R}^{\exp }\right),
$$

where $\sigma^{2}$ is the full covariance matrix made up of two terms, $\sigma^{2}=\sigma_{\text {unc }}^{2}+\sigma_{\text {cor }}^{2}$. The diagonal matrix $\sigma_{\text {unc }}^{2}$ contains the theoretical, statistical, and uncorrelated errors while $\sigma_{\text {cor }}^{2}$ contains the correlated systematic uncertainties. The $\mathbf{R}^{\mathrm{th} \text {,exp }}$ are length 2 vectors containing the theoretical and experimental data normalized to the SSM expectations. The $2 \times 2$ correlation matrix has been computed using standard techniques $[33,34]$. We have used data on the total event rates measured at the Homestake experiment, at the gallium experiments SAGE [14,35], GNO [36], and GALLEX [37] (see Table I for an explicit list of results and references). For the purposes of this work it is enough to summarize all the gallium experiments in one single quantity by taking the weighted average of their rates.

For the analysis of the SK energy spectrum, following closely the procedure assumed by the SK Collaboration $[32,38]$, we consider a $\chi^{2}$ function:

$$
\begin{aligned}
\chi_{\text {spec }}^{2}= & \sum_{d, n}\left(\alpha \mathbf{R}^{\text {th }}-\mathbf{R}^{\exp }\right)^{t}\left(\sigma_{\text {unc }}^{2}+\delta_{\text {cor }} \sigma_{\text {cor }}^{2}\right)^{-1} \\
& \times\left(\alpha \mathbf{R}^{\text {th }}-\mathbf{R}^{\exp }\right)+\chi_{\delta}^{2},
\end{aligned}
$$

where the vectors of data and expectations $\mathbf{R}$ are defined as before. We have introduced the SNO NC flux normalization factor $\alpha$ given above and the correlation parameter $\delta_{\text {cor }}$ is assumed to be constrained by the last term in the sum: $\chi_{\delta}^{2}$ $=\left(\delta_{\text {cor }}-\delta_{\text {cor }}^{\text {th }}\right)^{2} / \sigma_{\delta}^{2}$. The complete variance matrix is not a constant quantity. It is obtained from combining the statistical variances with systematic uncertainties and dependent on this correlation parameter. For each day and night spectrum the corresponding $19 \times 19$ block correlation matrices are conservatively constructed assuming full correlation among energy bins. The components of the variance matrix are given by standard expressions [15] in terms of statistic errors, bincorrelated and uncorrelated uncertainties. The data and errors

\begin{tabular}{|c|c|c|c|c|}
\hline $\begin{array}{l}\text { (A) Minimum LMA } \\
\text { (B) From Fit }\end{array}$ & $\begin{array}{c}\Delta m^{2}=5.44 \times 10^{-5} \mathrm{eV}^{2} \\
\Delta m^{2}=4.5_{-1.4}^{+2.7} \times 10^{-5} \mathrm{eV}^{2}\end{array}$ & $\begin{array}{c}\tan ^{2} \theta=0.40 \\
\tan ^{2} \theta=0.40_{-0.08}^{+0.10}\end{array}$ & $\chi_{m}^{2}=30.8$ & g.o.f.: $80 \%$ \\
\hline
\end{tabular}
for individual energy bins for the SK spectrum has been

TABLE II. Mixing parameters: (A) Best fit in the LMA region and (B) from fit to marginal likelihood distributions.

Method 
obtained from Ref. [32]. Other information from the SK results, such as the global day-night asymmetries, is to a large extent already contained in the above-mentioned quantities. It is therefore not included in our analysis and does not change the results presented further on.

\section{RESULTS AND DISCUSSION}

To test a particular oscillation hypothesis against the parameters of the best fit and obtain allowed regions in parameter space we perform a minimization of the fourdimensional function $\chi^{2}\left(\Delta m^{2}, \tan ^{2} \theta, \alpha, \delta_{\text {cor }}\right)$. For $\delta_{\text {cor }}$ $=\delta_{\mathrm{cor}}^{\min }, \alpha=\alpha_{\min }$, a given point in the oscillation parameter space is allowed if the globally subtracted quantity fulfills the condition $\Delta \chi^{2}=\chi^{2}\left(\Delta m^{2}, \theta\right)-\chi_{\min }^{2}<\chi_{n}^{2}$ (C.L.). Where $\chi_{n=4}^{2}(90 \%, 95 \%, \ldots)=7.78,9.4, \ldots$ are the quantiles for four degrees of freedom. The $\chi^{2}$ summation now contains 41 bins in total: 3 from the global rates and SNO asymmetry and $2 \times 19$ bins for the SK day and night spectrums.

The results are shown in Fig. 1 (left) where we have generated acceptance contours in $\Delta m^{2}$ and $\tan ^{2} \theta$. In Table II we present the best fit parameters or local minima obtained from the minimization of the $\chi^{2}$ function given in Eq. (3). Also shown are the values of $\chi_{\min }^{2}$ per degree of freedom $\left(\chi^{2} / n\right)$ and the goodness of fit (g.o.f.) or significance level of each point (definition of SL as in Ref. [39]). In Fig. 1 (right) we superimpose the global signal expected in the KamLAND experiment from reactor electron antineutrinos. We observe that at the most favored regions obtained before, the KamLAND expected signal is situated at a intermediate region where high sensibility to both mass squared and mixing angle parameters is found. The experiment expects around $50 \%$ of the nonoscillation signal, in this region it would suffice to reach a total error of 5\%-10\% (a quantity reachable after 1 year of data taking) in the measurement to be able to confirm the SNO results.

In order to obtain concrete values for the individual oscillation parameters and estimates for their uncertainties, it is preferable to study the marginalized parameter constraints. It is justified to convert $\chi^{2}$ into likelihood using the expression $\mathcal{L}=e^{-\chi^{2} / 2}$, this normalized marginal likelihood is plotted in Fig. 2 for each of the oscillation parameters $\Delta m^{2}$ and $\tan ^{2} \theta$. For $\tan ^{2} \theta$ we observe that the likelihood function is concentrated in a region $0.2<\tan ^{2} \theta<1$ with a clear maximum at $\tan ^{2} \theta \sim 0.4$. The situation for $\Delta m^{2}$ is similar. Values for the parameters are extracted by fitting one- or two-sided Gaussian distributions to any of the peaks (fits not shown in the plots). In the case of the angle distribution the goodness of fit of the Gaussian fit is excellent (g.o.f $>99.9 \%$ ) even at far
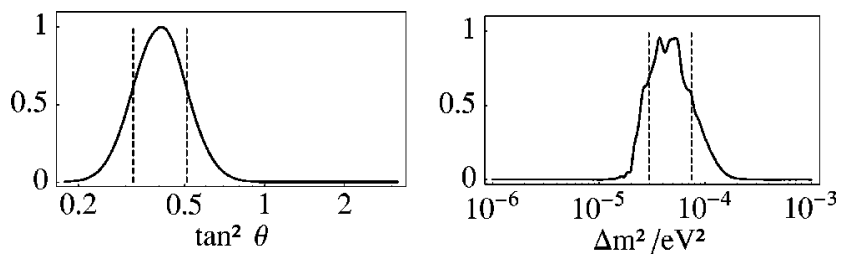

FIG. 2. Marginalized likelihood distributions for each of the oscillation parameters $\Delta m^{2}$ (right), $\tan ^{2} \theta$ (left). The curves are in arbitrary units with normalization to the maximum height. Values for the peak position are obtained by fitting two-sided Gaussian distrbutions (not shown in the plot). Dashed lines delimit $\pm 1 \sigma$ error regions around the maximum.

tail distances thus justifying the consistency of the procedure. The goodness of Gaussian fit to the distribution in squared mass, although somewhat smaller, is still good. The values for the parameters appear in Table II. They are fully consistent and very similar to the values obtained from simple $\chi^{2}$ minimization.

\section{CONCLUSIONS}

In summary, in this work we have presented an up-to-date analysis of all available solar neutrino evidence including the latest SNO results in the most simple framework. The direct measurement via the $\mathrm{NC}$ reaction on deuterium of ${ }^{8} \mathrm{~B}$ neutrinos combined with the $\mathrm{CC}$ results have largely confirmed the neutrino oscillation hypothesis. We have obtained the allowed area in parameter space and individual values for $\Delta m^{2}$ and $\tan ^{2} \theta$ with error estimation from the analysis of marginal likelihoods. In the framework of two active neutrino oscillations we obtain

$$
\Delta m^{2}=4.5_{-1.4}^{+2.7} \times 10^{-5} \mathrm{eV}^{2}, \quad \tan ^{2} \theta=0.40_{-0.08}^{+0.10} .
$$

We have shown that it is already possible to determine at present active two neutrino oscillation parameters with relatively good accuracy. The KamLAND experiment (see Ref. [40] and specially the near future long baseline experiments will have a clear chance of first confirming present mixing parameters obtained from solar originated neutrinos, and second, measuring first and second generation mass and mixing parameters under laboratory-controlled conditions.

\section{ACKNOWLEDGMENTS}

We acknowledge the financial support of the Italian MIUR, the Spanish CYCIT funding agencies, and the CERN Theoretical Division. The numerical calculations have been performed in the computer farm of the Milano University theoretical group.
[1] SNO Collaboration, Q. R. Ahmad et al., Phys. Rev. Lett. 89, 011301 (2002).

[2] SNO Collaboration, Q. R. Ahmad et al., Phys. Rev. Lett. 89, 011302 (2002).

[3] S. Turck-Chieze, Nucl. Phys. B (Proc. Suppl.) 91, 73 (2001);
E. G. Adelberger et al., Rev. Mod. Phys. 70, 1265 (1998); A. S. Brun, S. Turck-Chieze, and P. Morel, astro-ph/9806272.

[4] J. N. Bahcall, M. H. Pinsonneault, and S. Basu, Astrophys. J. 555, 990 (2001).

[5] J. N. Bahcall and M. H. Pinsonneault, Rev. Mod. Phys. 67, 781 
(1995).

[6] SNO Collaboration, J. R. Klein, in Proceedings of the 8th International Workshop on Neutrino Telescopes, Venice, 1999, Vol. 1, pp. 115-125; SNO Collaboration, A. B. McDonald, Nucl. Phys. B (Proc. Suppl.) 77, 43 (1999).

[7] SNO Collaboration, J. Boger et al., Nucl. Instrum. Methods Phys. Res. A 449, 172 (2000).

[8] V. Barger, D. Marfatia, and K. Whisnant, Phys. Lett. B 509, 19 (2001).

[9] J. N. Bahcall, P. I. Krastev, and A. Y. Smirnov, J. High Energy Phys. 05, 015 (2001).

[10] SNO Collaboration, Q. R. Ahmad et al., Phys. Rev. Lett. 87, 071301 (2001).

[11] R. Davis, Prog. Part. Nucl. Phys. 32, 13 (1994); Homestake Collaboration, B. T. Cleveland et al., Nucl. Phys. B (Proc. Suppl.) 38, 47 (1995); Homestake Collaboration, B. T. Cleveland et al., Astrophys. J. 496, 505 (1998).

[12] Super-Kamiokande Collaboration, Y. Fukuda et al., Phys. Rev. Lett. 82, 1810 (1999).

[13] Super-Kamiokande Collaboration, Y. Fukuda et al., Phys. Rev. Lett. 81, 1158 (1998); 81, 4279(E) (1998).

[14] SAGE Collaboration, J. N. Abdurashitov et al., Phys. Rev. Lett. 83, 4686 (1999).

[15] P. Aliani, V. Antonelli, M. Picariello, and E. Torrente-Lujan, Nucl. Phys. B (Proc. Suppl.) 110, 361 (2002); Nucl. Phys. B634, 393 (2002); E. Torrente-Lujan, Phys. Rev. D 53, 4030 (1996); P. Aliani, V. Antonelli, R. Ferrari, M. Picariello, and E. Torrente-Lujan, hep-ph/0206308; hep-ph/0205061.

[16] Super-Kamiokande Collaboration, Y. Fukuda et al., Phys. Rev. Lett. 82, 1810 (1999); J. N. Bahcall, P. I. Krastev, and E. Lisi, Phys. Rev. C 55, 494 (1997); J. N. Bahcall and E. Lisi, Phys. Rev. D 54, 5417 (1996); J. N. Bahcall, E. Lisi, D. E. Alburger, L. De Braeckeleer, S. J. Freedman, and J. Napolitano, Phys. Rev. C 54, 411 (1996).

[17] V. Barger, D. Marfatia, and K. Whisnant, Phys. Rev. Lett. 88, 011302 (2002).

[18] G. L. Fogli, E. Lisi, D. Montanino, and A. Palazzo, Phys. Rev. D 64, 093007 (2001).

[19] P. I. Krastev and A. Y. Smirnov, Phys. Rev. D 65, 073022 (2002)

[20] J. N. Bahcall, M. C. Gonzalez-Garcia, and C. Pena-Garay, J. High Energy Phys. 08, 014 (2001); 04, 007 (2002).

[21] A. Bandyopadhyay, S. Choubey, S. Goswami, and K. Kar, Phys. Rev. D 65, 073031 (2002).

[22] S. Choubey, S. Goswami, and D. P. Roy, Phys. Rev. D 65, 073001 (2002).

[23] A. Bandyopadhyay, S. Choubey, S. Goswami, and K. Kar, Phys. Lett. B 519, 83 (2001).

[24] E. Torrente-Lujan, Phys. Rev. D 59, 093006 (1999); 59, 073001 (1999); Phys. Lett. B 441, 305 (1998); V. B. Semikoz and E. Torrente-Lujan, Nucl. Phys. B556, 353 (1999); E. Torrente-Lujan, Phys. Lett. B 494, 255 (2000); hep-ph/
9902339; S. Khalil and E. Torrente-Lujan, J. Egypt. Math. Soc. 9, 91 (2001).

[25] I. Mocioiu and R. Shrock, Phys. Rev. D 62, 053017 (2000); A. Dziewonski, in The Encyclopedia of Solid Earth Geophysics, edited by D. E. James (Van Nostrand Reinhold, New York, 1989).

[26] J. Bahcall, M. Kamionkowski, and A. Sirlin, Phys. Rev. D 51, 6146 (1995).

[27] S. Nakamura, T. Sato, V. Gudkov, and K. Kubodera, Phys. Rev. C 63, 034617 (2001).

[28] SNO Collaboration, Q. R. Ahmad et al., "HOWTO use the SNO Solar Neutrino Spectral data," http://www.sno.phy.queensu.ca/sno

[29] Super-Kamiokande Collaboration, M. Nakahata et al., Nucl. Instrum. Methods Phys. Res. A 421, 113 (1999).

[30] H. Ishino, Ph.D. thesis, University of Tokyo, 1999; SuperKamiokande Collaboration, M. Nakahata et al., Nucl. Instrum. Methods Phys. Res. A 421, 113 (1999).

[31] N. Sakurai, Ph.D. thesis, 2000.

[32] Super-Kamiokande Collaboration, S. Fukuda et al., Phys. Rev. Lett. 86, 5651 (2001).

[33] G. L. Fogli and E. Lisi, Astropart. Phys. 3, 185 (1995).

[34] S. Goswami, D. Majumdar, and A. Raychaudhuri, Phys. Rev. D 63, 013003 (2001).

[35] SAGE Collaboration, A. I. Abazov et al., Phys. Rev. Lett. 67, 3332 (1991); SAGE Collaboration, D. N. Abdurashitov et al., ibid. 77, 4708 (1996); SAGE Collaboration, J. N. Abdurashitov et al., Phys. Rev. C 60, 055801 (1999); SAGE Collaboration, J. N. Abdurashitov et al., Phys. Rev. Lett. 83, 4686 (1999); SAGE Collaboration, J. N. Abdurashitov et al., Phys. Rev. C 60, 055801 (1999).

[36] GNO Collaboration, M. Altmann et al., Phys. Lett. B 490, 16 (2000).

[37] GALLEX Collaboration, P. Anselmann et al., Phys. Lett. B 285, 376 (1992); GALLEX Collaboration, W. Hampel et al., ibid. 388, 384 (1996); T. A. Kirsten, Prog. Part. Nucl. Phys. 40, 85 (1998); GALLEX Collaboration, W. Hampel et al., Phys. Lett. B 447, 127 (1999); M. Cribier, Nucl. Phys. B (Proc. Suppl.) 70, 284 (1999); GALLEX Collaboration, W. Hampel et al., Phys. Lett. B 436, 158 (1998); GALLEX Collaboration, W. Hampel et al., ibid. 447, 127 (1999).

[38] Super-Kamiokande Collaboration, S. Fukuda et al., Phys. Rev. Lett. 86, 5656 (2001).

[39] Particle Data Group, D. E. Groom et al., Eur. Phys. J. C 15, 1 (2000).

[40] P. Aliani, V. Antonelli, M. Picariello, and E. Torrente-Lujan, hep-ph/0208089; hep-ph/0207348.

[41] For the Homestake Collaboration, K. Lande, Nucl. Phys. B (Proc. Suppl.) 77, 13 (1999).

[42] CHOOZ Collaboration, M. Apollonio et al., Phys. Lett. B 466, 415 (1999); 420, 397 (1998); F. Boehm et al., Phys. Rev. D 62, 072002 (2000). 